\title{
Laser sources for precision spectroscopy on atomic strontium
}

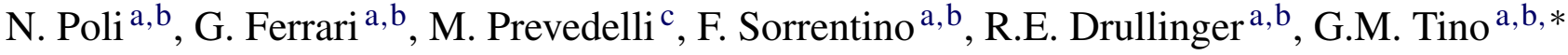 \\ ${ }^{a}$ Dipartimento di Fisica and LENS, Instituto Nazionale di Fisica Nucleare, Sezione di Firenze, Italy \\ ${ }^{\mathrm{b}}$ Instituto Nazionale di Fisica della Materia, Unità di Firenze, Polo Scientifico, 50019 Sesto Fiorentino, Italy \\ c Dipartimento di Chimica Fisica, Università di Bologna, Via del Risorgimento 4, 40136 Bologna, Italy
}

Received 15 September 2005; received in revised form 2 October 2005; accepted 9 October 2005

\begin{abstract}
We present a new laser setup designed for high-precision spectroscopy on laser cooled atomic strontium. The system, which is entirely based on semiconductor laser sources, delivers $200 \mathrm{~mW}$ at $461 \mathrm{~nm}$ for cooling and trapping atomic strontium from a thermal source, $4 \mathrm{~mW}$ at $497 \mathrm{~nm}$ for optical pumping from the metastable ${ }^{3} \mathrm{P}_{2}$ state, $12 \mathrm{~mW}$ at $689 \mathrm{~nm}$ on linewidth less than $1 \mathrm{kHz}$ for second-stage cooling of the atomic sample down to the recoil limit, $1.2 \mathrm{~W}$ at $922 \mathrm{~nm}$ for optical trapping close to the "magic wavelength" for the $0-1$ intercombination line at $689 \mathrm{~nm}$. The $689 \mathrm{~nm}$ laser was already employed to perform a frequency measurement of the $0-1$ intercombination line with a relative accuracy of $2.3 \times 10^{-11}$, and the ensemble of laser sources allowed the loading in a conservative dipole trap of multi-isotopes strontium mixtures. The simple and compact setup developed represents one of the first steps towards the realization of a transportable optical standards referenced to atomic strontium.

(C) 2005 Elsevier B.V. All rights reserved.
\end{abstract}

PACS: 42.55.Px Semiconductor lasers; Laser diodes 42.65.Ky Frequency conversion; Harmonic generation, including higher order harmonic generation, 32.80.Pj Optical cooling of atoms; Trapping

Keywords: Laser diodes; Non-linear optics; Optical cooling of atoms; Isotopes; BEC

\section{Introduction}

Strontium has become the object of recent study in atomic physics ranging from frequency metrology [1] to cold collisions [2] and multiple scattering [3].

The level scheme (see Fig. 1) allows the possibility to directly cool strontium with standard Doppler cooling techniques to temperatures well below $1 \mu \mathrm{K}$ using two steps of trapping and cooling in magneto-optical trap working on the dipole allowed ${ }^{1} \mathrm{~S}_{0}-{ }^{1} \mathrm{P}_{1}$ at $461 \mathrm{~nm}$ and on the ${ }^{1} \mathrm{~S}_{0}-{ }^{3} \mathrm{P}_{1}$ transition. As a result of the efficient double-stage Doppler cooling [4], strontium is a promising candidate to reach quantum degeneracy. Secondstage Doppler cooling working on the ${ }^{1} \mathrm{~S}_{0}-{ }^{3} \mathrm{P}_{1}$ intercombination transition (see Fig. 1) combined with optical dipole trapping was proven effective to directly reach phase-space densities of $\rho=0.1$ [5]. Further increase of $\rho$ is then limited by light-assisted collisions, thus alternative cooling techniques have to be applied, like evaporative cooling or sympathetic cooling through different atoms or isotopic mixtures. A degenerate sample of strontium

\footnotetext{
* Corresponding author.

E-mail address: Guglielmo.Tino@fi.infn.it (G.M. Tino).
}

would represent an interesting system, and may open the way to novel applications in matter-wave interferometry and quantum sensors.

Thanks to the ultra-narrow intercombination transitions, strontium is also the object of intense study in the field of optical frequency metrology. These studies open the way to the realization of a new optical frequency standard with unprecedented stability and accuracy. Proposals based on different interrogation schemes have been put forward (optical lattice clock [6], engineered atom clock $[7,8]$ ) suggesting the possibility to reach the $10^{-17}$ level of overall accuracy. Indeed, thanks to the higher oscillator frequency, a clock in the optical domain could potentially reach a stability level of $10^{-18}$ at $1 \mathrm{~s}[6]$ which represents a factor of $10^{4}$ improvement with respect to the best microwave standards [9]. A clock with such stability and accuracy could be used as a powerful test for relativistic theory, searching for deviation of fine structure constant and to define a new atomic time standard. The high stability would find direct application in deep space navigation, remote sensing, secure telecommunications and ultra-precise geodesy.

At present, our work is progressing along two parallel avenues: (1) trapping and cooling of strontium and (2) working toward a suitable local oscillator for an optical clock based on 


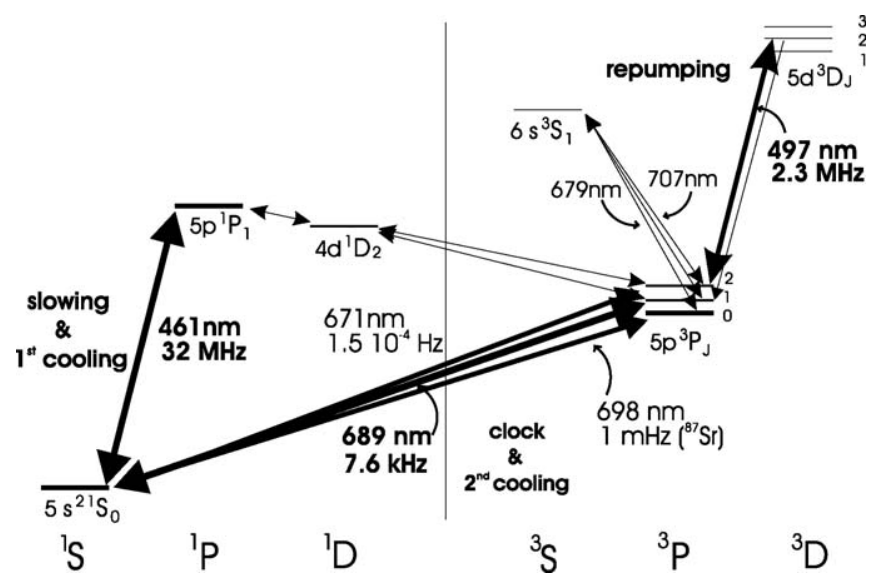

Fig. 1. Simplified energy levels and optical transition for strontium.

optical intercombination transitions of strontium. The structure of the article is the following. Section 2 describes the stable red source at $689 \mathrm{~nm}$ used for absolute frequency measurements of intercombination lines ${ }^{1} \mathrm{~S}_{0}-{ }^{3} \mathrm{P}_{1}$ of strontium and for secondstage Doppler cooling on the same transition. In sections 3, 4 and 5, we present the blue laser at $461 \mathrm{~nm}$ employed in the first cooling stage on the ${ }^{1} \mathrm{~S}_{0}-{ }^{1} \mathrm{P}_{1}$ transition, the green laser at $497 \mathrm{~nm}$ for repumping on the ${ }^{3} \mathrm{P}_{2}-{ }^{3} \mathrm{D}_{2}$ transition and the optical dipole trapping laser, based on $922 \mathrm{~nm}$ infrared sources. Section 6 is dedicated to the absolute frequency measurements of the ${ }^{1} \mathrm{~S}_{0}-$ ${ }^{3} \mathrm{P}_{1}$ intercombination lines for ${ }^{88} \mathrm{Sr}$ and ${ }^{86} \mathrm{Sr}$, finally, Section 7 describes some preliminary results on laser cooling and trapping of strontium atoms.

\section{Stable laser at $689 \mathrm{~nm}$}

The scheme of our stable master source resonant with the intercombination ${ }^{1} \mathrm{~S}_{0}-{ }^{3} \mathrm{P}_{1}$ transition for the bosonic strontium isotope is reported in Fig. 2. The master laser is a $689 \mathrm{~nm}$ diode laser mounted in extended cavity configuration (ECDL, Littrow mount). The reduction of the fast linewidth is realized by locking the laser to a resonance of a reference cavity with standard Pound-Drever-Hall technique [10]. The laser electric field is phase modulated at $11 \mathrm{MHz}$ with an electro-optic modulator (EOM) with a modulation index $m<1$, while the error signal is derived by demodulating the light signal reflected from the cavity. In order to reduce the feedback light from the cavity going back to the laser, we use an optical isolator and an acousto-optic modulator (AOM) in cascade at the output of the laser.

The cavity is composed by two mirrors (99.95\% reflectivity at $689 \mathrm{~nm})$, a plane and a curved one $(r=50 \mathrm{~cm})$ and a $10 \mathrm{~cm}$ quartz spacer. The cavity is placed on a massive iron V-block (20 kg approx.) aligned along the horizontal axis. The vacuum chamber that contains the cavity and the iron block is evacuated $\left(10^{-6} \mathrm{~Pa}\right)$ and the vacuum is maintained with a $21 / \mathrm{s}$ ion pump. In order to attenuate vibration from the optical table induced into the cavity, pieces of Viton O-ring and rubber are used between the cavity and the iron and between the iron and the vacuum cell. The optical table is also supported with an active air damping system.

The reflected light from the cavity is collected with a $\mathrm{Si}$ photodiode, amplified with a fast and low current noise transimpedance amplifier and finally down-converted with a mixer. The level of the voltage noise at the output of the detector is $-110 \mathrm{dBV} / \sqrt{\mathrm{Hz}}$ which corresponds to the shot noise of $100 \mu \mathrm{W}$ of incident light. The cavity resonance has a FWHM of $170 \mathrm{kHz}$. In this condition the slope of the error signal is $3.4 \mathrm{~V} / \mathrm{MHz}$. The error signal has then $\mathrm{S} / \mathrm{N} \sim 200$ on a bandwidth of $3 \mathrm{MHz}$. The error signal for locking the laser to the cavity is then sent to the laser via three channels: (1) to the PZT of the external cavity of the laser with a bandwidth up to $2 \mathrm{kHz}$; (2) to the laser current driver with a bandwidth up to $50 \mathrm{kHz}$; (3) directly to the diode with a bandwidth up to $3 \mathrm{MHz}$.

The signal to noise ratio in our case would be sufficient to achieve laser linewidth of less than $1 \mathrm{~Hz}$. Actually the laser linewidth is limited by noise induced in the cavity (mainly acoustic and sub-acoustic vibration) which is then transferred as frequency fluctuations directly to the laser. By using a resonance mode of a second cavity as a frequency discriminator, we could determine the absolute frequency noise of our master laser. This cavity has the same geometry of the first cavity, it is placed on the same optical table with its horizontal axis orthogonally (a)

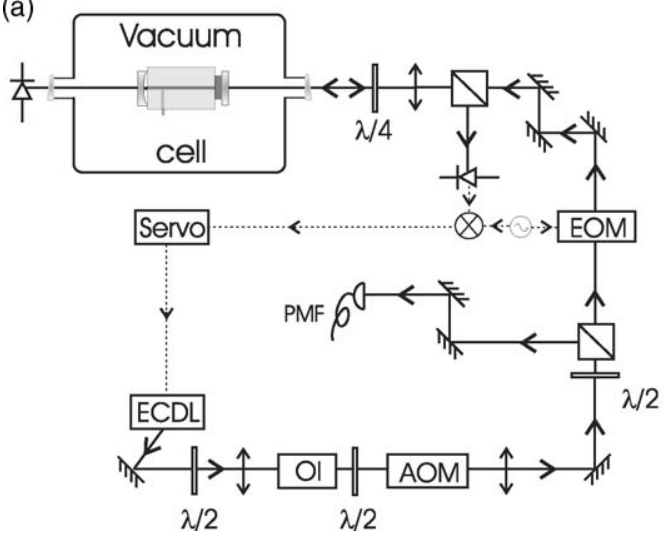

(b)

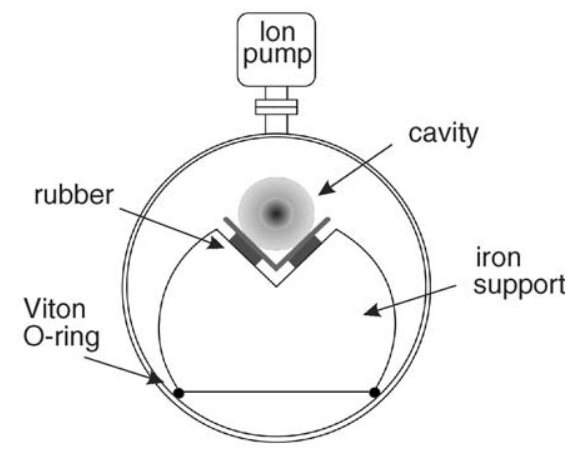

Fig. 2. (a) Experimental setup for the stable $689 \mathrm{~nm}$ laser, ECDL: extended cavity diode laser, AOM: acousto-optic modulator, OI: $30 \mathrm{~dB}$ optical isolator, EOM: electro-optic modulator, PMF: polarization maintaining fiber. (b) Detail of the cavity suspension; the diameter of the outside cell is $20 \mathrm{~cm}$. 


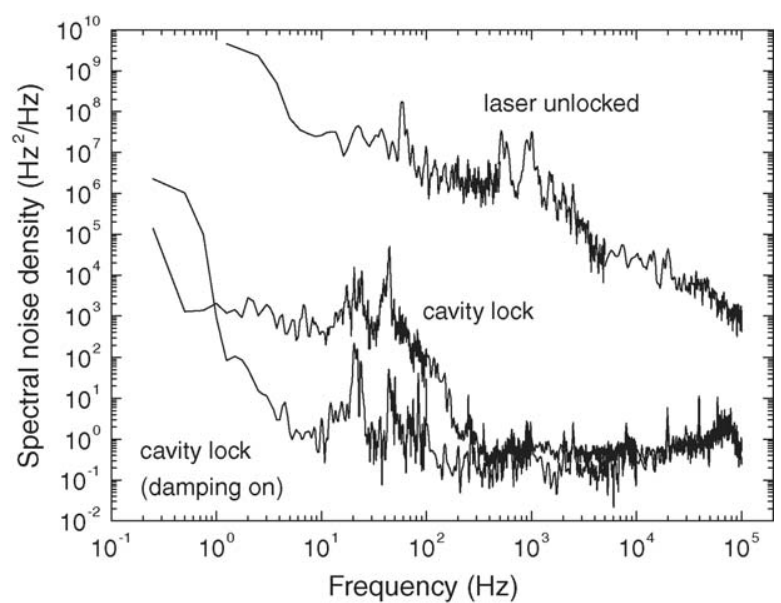

Fig. 3. Frequency noise of the laser locked to the cavity. From the lower line we can infer a laser linewidth of about $20 \mathrm{~Hz}$.

aligned with respect to the first cavity. The measurements are reported in Fig. 3 respectively for laser unlocked, laser locked to the cavity, and laser locked to the cavity with the air damping working.

From the integrated noise measured on the second cavity we can infer a fast linewidth $\Delta v \sim 20 \mathrm{~Hz}$, mainly limited by some excess noise peaked around $20 \mathrm{~Hz}$ and $40 \mathrm{~Hz}$. While the resulting laser linewidth is sufficiently narrow for doing spectroscopy on the broad ${ }^{1} \mathrm{~S}_{0^{-}}{ }^{3} \mathrm{P}_{1}$ line for ${ }^{88} \mathrm{Sr}$, for spectroscopy on ultranarrow strontium transitions we are setting up a second stage of stabilization to a higher finesse cavity sustained with a better isolation from acoustic noise.

For the long term stability of the laser we actively stabilize the length of the reference cavity with a lock to an atomic signal coming from saturation spectroscopy on a strontium heat pipe. For this purpose we use a PZT transducer, placed between the flat mirror of the cavity and the quartz spacer, for tuning the cavity length (PZT sensitivity $38 \mathrm{MHz} / \mathrm{V})$. In order to reduce electronic noise induced by the PZT, two different voltages are applied to the two side of the PZT transducer: the first for coarse tuning of the cavity (up to $2 \mathrm{GHz}$ ) comes from a battery, while the small cavity length correction (up to $2 \mathrm{MHz}$ ) is delivered from low noise electronics.

The heat pipe is made up with a $2.5 \mathrm{~cm}$ diameter tube with a length of $60 \mathrm{~cm}$. The central part is filled with metallic strontium and is maintained at $500{ }^{\circ} \mathrm{C}$. At both ends of the tube, BK7 windows, glued at Brewster angle, provide the optical access. In order to prevent chemical attack or condensation of the strontium vapour on the window, a buffer gas (He or Ar) is loaded at a pressure of $10^{-2}$ mbar and the tube is water cooled on both sides. The mean free path of strontium atoms in this condition is of the order of $1 \mathrm{~cm}$, less than the distance to the window from the hot center, while the linewidth we expect from pressure broadening is about $30 \mathrm{kHz}$ [11].

In the heat pipe we do a pump-probe spectroscopy. To avoid interference between probe and pump beams, the pump beam is shifted of $160 \mathrm{MHz}$ with a double pass AOM, which is also used for the low-frequency modulation $(\sim 10 \mathrm{kHz})$ of the pump beam. The two beams have similar diameter of about $1 \mathrm{~cm}$ and a power respectively of 200 and $50 \mu \mathrm{W}$. The pump beam absorption on resonance is about $10 \%$. The quantization axis is defined parallel to the polarization axis of the laser beams applying a magnetic field of $\mathrm{B} \sim 10 \mathrm{mT}$ with four permanent magnets. The sub-Doppler signal obtained has a FWHM of $200 \mathrm{kHz}$ mainly limited by power broadening. This signal is then demodulated with the low frequency modulation used for the pump beam and the error signal obtained has typically a $\mathrm{S} / \mathrm{N}$ ratio of 60 in a $10 \mathrm{~Hz}$ bandwidth. This $\mathrm{S} / \mathrm{N}$ ratio is enough in order to lock the laser to the line center with an uncertainty of $2 \mathrm{kHz}$.

The drift of the cavity in normal operation is of the order of $20 \mathrm{MHz} / \mathrm{h}$. In order to correct for this slow drift, we send the integrated error signal to the PZT of the cavity with an overall bandwidth of about $50 \mathrm{~Hz}$. In normal operation condition, the dynamic range of the servo electronics assures a stable lock for about a day. The overall system (master laser, vacuum cell and optics) is quite compact and it fits on a single $60 \mathrm{~cm} \times 60 \mathrm{~cm}$ aluminium breadboard.

\section{Blue laser source at $461 \mathrm{~nm}$}

For first cooling and trapping strontium atoms on ${ }^{1} \mathrm{~S}_{0}-{ }^{1} \mathrm{P}_{1}$ transition $\left(\Gamma / 2 \pi=32 \mathrm{MHz}, I_{\text {sat }}=42 \mathrm{~mW} / \mathrm{cm}^{2}\right)$ an intense $(>$ $100 \mathrm{~mW}$ ) source of $461 \mathrm{~nm}$ blue light is needed. For this purpose, we developed an all-solid-state laser source based on a master ECDL at $922 \mathrm{~nm}$, amplified in a semiconductor tapered amplifier (TA) and then frequency doubled with a periodically poled KTP crystal, placed in an optical resonator. A scheme of the laser setup is reported in Fig. 4. The light from the ECDL (typically $50 \mathrm{~mW}$ ) is sent to the TA through a $60 \mathrm{~dB}$ optical isolator. The TA is based on a chip mounted on a temperature stabilized holder which includes the optics for injection and output collimation. Injecting the TA with $40 \mathrm{~mW}$ and driving $3.4 \mathrm{~A}$ we obtain typically $800 \mathrm{~mW}$ after a $35 \mathrm{~dB}$ optical isolator, spatial mode filtering through a pinhole, and the beam shaping optics for the coupling into the doubler resonator. Typically, $60 \%$ of this light is then sent to the doubling cavity, while the rest is used to inject other TA used for dipole trapping beams.

The frequency doubler is composed of a non-linear crystal (25 mm long periodically poled KTP crystal) placed in an optical build up cavity. The crystal facets are anti-reflection coated both at 922 and $461 \mathrm{~nm}(R<0.2 \%)$ and the poling period is chosen to fulfill the quasi-phase-matching of our wavelength at room temperature. For an optimal waist of $w=26 \mu \mathrm{m}$, we obtain a maximum single pass efficiency of $\gamma=4.8 \% \mathrm{~W}^{-1}$ at room temperature.

The resonator has an input coupler with $11 \%$ transmission and it is held in resonance with the input light feeding the error signal from a Hänsch-Couillaud detection [12] to a PZT controlled folding mirror. Under optimal conditions we obtain $200 \mathrm{~mW}$ in the blue and routinely we work with $130 \mathrm{~mW}$. The overall efficiency of $40 \%$ is explained by excess linear losses inside the resonator.

The source is then frequency referenced to the atomic line by locking the master infrared laser with an error signal obtained 


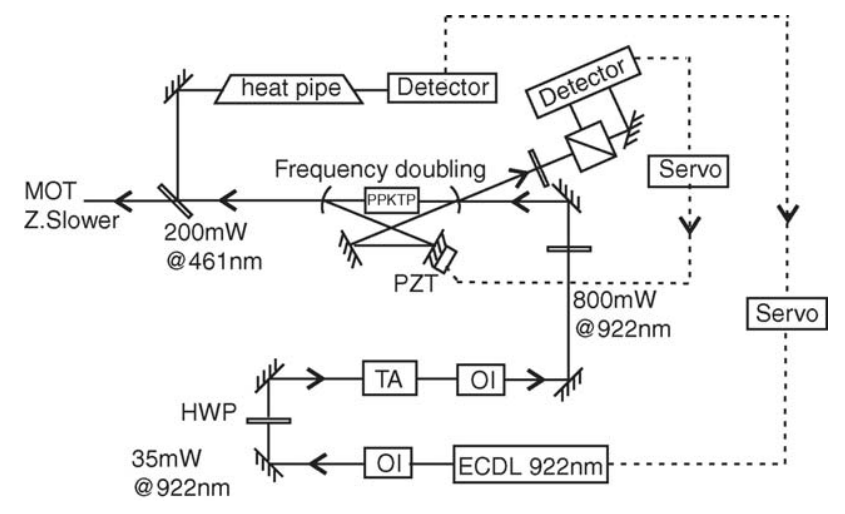

Fig. 4. Setup of the blue laser source: the light from an extended cavity diode laser (ECDL) is amplified on a semiconductor tapered amplifier (TA) and then it is frequency doubled on a periodically poled KTP crystal (PPKTP) placed in an enhancement cavity. The optical cavity is kept on resonance with the incoming light with a Hänsch-Couillaud lock, while the frequency of the ECDL is kept on resonance with the ${ }^{1} \mathrm{~S}_{0}-{ }^{1} \mathrm{P}_{1}$ transition by saturation spectroscopy on a strontium heat pipe. BSO: beam shaping optics. WP: wave plate. HWP: half-wave plate. HP: heat pipe.

from saturation spectroscopy on a second strontium heat pipe [13].

\section{Green laser source at $497 \mathrm{~nm}$}

During the first cooling stage on ${ }^{1} \mathrm{~S}_{0}-{ }^{1} \mathrm{P}_{1}$ transition at $461 \mathrm{~nm}$, a fraction of the atoms falls into the dark ${ }^{3} \mathrm{P}_{2}$ metastable state due to the $2 \times 10^{-5}$ branching ratio of the ${ }^{1} \mathrm{P}_{1}$ excited state to the low-lying ${ }^{1} \mathrm{D}_{2}$, that subsequently decays to the ${ }^{3} \mathrm{P}_{2}$ state. This loss channel can be eliminated with a single optical pumping process, which involves the ${ }^{3} \mathrm{P}_{2}-{ }^{3} \mathrm{D}_{2}$ transition at $497 \mathrm{~nm}$ [14]. Thanks to the low saturation intensity $\left(I_{\mathrm{sat}}=2.4 \mathrm{~mW} / \mathrm{cm}^{2}\right)$ we need only some $\mathrm{mW}$ at this color.

For this purpose, we developed a laser source based on an antireflection coated laser diode stabilized in the Littrow extended cavity configuration, which delivers $35 \mathrm{~mW}$ at $994 \mathrm{~nm}$. This light is frequency doubled on a 17-mm-long bcut $\mathrm{KNbO}_{3}$ crystal placed in a resonant cavity. Non critical phase-matching condition is then obtained by temperature tuning the crystal to a temperature of $56^{\circ} \mathrm{C}$. A maximum single pass efficiency for this crystal of $\gamma=6.1 \% \mathrm{~W}^{-1}$ is obtained for incident beam waist of $w=21 \mu \mathrm{m}$. With an $8 \%$ transmission input coupler, about $60 \%$ of the incident light is coupled into the resonant cavity, producing a maximum total efficiency of $\eta=13 \%$, resulting in $4 \mathrm{~mW}$ of green light at $497 \mathrm{~nm}$.

The $497 \mathrm{~nm}$ beam is then sent to the MOT region with a $1.5 \mathrm{~cm} 1 / e^{2}$ diameter, it is back-reflected, and its frequency is tuned in order to maximize the blue MOT fluorescence.

\section{Infrared sources at $922 \mathrm{~nm}$ for dipole trapping}

After the production of ultracold sample in two stages trapping, we transfer the atoms in a crossed dipole trap. The two beams, respectively aligned along the horizontal and vertical direction, are produced with two independent TA injected with

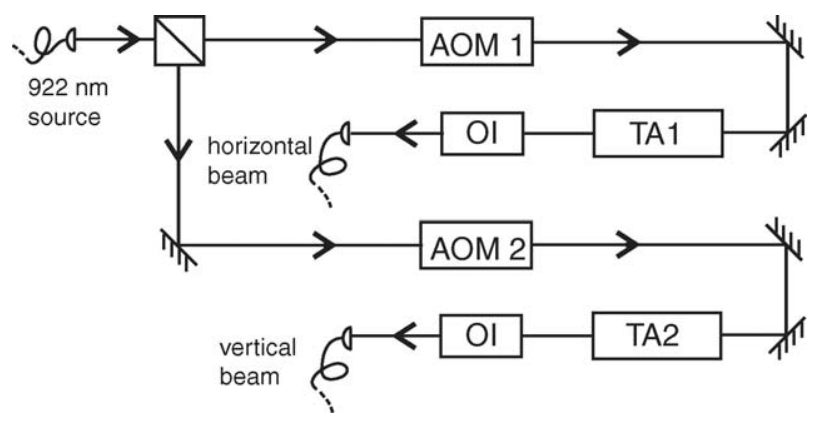

Fig. 5. Experimental setup of the infrared source used for the crossed dipole trap. The two beams are delivered with two independent tapered amplifiers injected with $922 \mathrm{~nm}$ light coming from the infrared source used for producing the blue light. Two AOMs before the injection are used for modulating the amplitude of the laser and to shift the two light frequencies. AOM acousto-optical modulator, TA tapered amplifier, OI optical isolator.

light coming from the first infrared source used for producing the blue light (see Fig. 5).

Typically $300 \mathrm{~mW}$ of light coming from that source are coupled into a fiber and sent to the two amplifiers. The injection of the two TAs is regulated by two AOMs which are used to shift the frequency of the two beams (avoiding interference at the center of the dipole trap) and to apply a fast control in output TA optical power. For mode cleaning and delivering the output beams from the TAs to the atoms, we use two independent single mode fibers. Typically, we obtain about $600 \mathrm{~mW}$ for each beam at the output of each fiber. Finally, the beams are focused in the center of the MOT with $1 / e^{2}$ radius of $w=35 \mu \mathrm{m}$. The depth of the crossed dipole trap potential is $U_{0}=7 \mu \mathrm{K}$ with a mean trap frequency $\bar{\omega}=315 \mathrm{~Hz}$. The wavelength used for the dipole trap is only $7 \mathrm{~nm}$ from the "magic wavelength" for the intercombination ${ }^{1} \mathrm{~S}_{0}-{ }^{3} \mathrm{P}_{1}$ transition [15]. Choosing the polarization of the dipole trapping light orthogonal to the magnetic field the Stark shift for the ${ }^{1} \mathrm{~S}_{0}-{ }^{3} \mathrm{P}_{1}$ transition is below to $10 \mathrm{kHz}$. Thus, allowing the possibility to cool the atoms during the loading of the dipole trap [5].

\section{Absolute frequency measurement}

In a previous measurement, before the operation of the MOT, we measured the absolute frequency of the ${ }^{1} \mathrm{~S}_{0}-{ }^{3} \mathrm{P}_{1}$ intercombination line at $689 \mathrm{~nm}$. For the absolute frequency measurement we used a fs-optical-comb-generator; details of the experimental apparatus can also be found in [16,17]. Part of the light coming from the pre-stabilized $689 \mathrm{~nm}$ source is coupled into a polarization maintaining fiber and delivered to a strontium thermal atomic beam, while a second light beam is sent towards the comb with a $20 \mathrm{~m}$ fiber. With standard saturation spectroscopy techniques we obtained a sub-Doppler signal used for long term stabilization of the length of the cavity. The values measured for the two most abundant isotopes are 434829121311 (10) $\mathrm{kHz}$ for ${ }^{88} \mathrm{Sr}$ and 434828957494 (10) $\mathrm{kHz}$ for ${ }^{86} \mathrm{Sr}$. The latter value has been derived by difference from the measurement of the ${ }^{88} \mathrm{Sr}-{ }^{86} \mathrm{Sr}$ isotopic shift (163 $817.4(0.2) \mathrm{kHz})$. In the absolute frequency measurement, one of the main limitations was found to be the instability of 
our GPS referenced quartz used as local oscillator for the comb [16].

\section{Cooling and trapping strontium}

The experimental setup used for trapping and cooling strontium atoms is based on a decelerated atomic beam; details of this setup can be found in [13]. The setup includes the oven (typically heated at $500^{\circ} \mathrm{C}$ ) which delivers an intense and highly collimated $(25 \mathrm{mrad})$ atomic beam thanks to the use of a bundle of capillaries, a transverse 2D optical molasses stage, which is used to further improve the collimation of the beam, a 30-cm Zeeman slower and the MOT cell. All the blue frequencies used for collimation, slowing and MOT beams are produced with AOMs that shift the frequency of the light coming from the main blue source to the red of the ${ }^{1} \mathrm{~S}_{0}-{ }^{1} \mathrm{P}_{1}$ transition respectively of $-20 \mathrm{MHz}$, $-480 \mathrm{MHz},-40 \mathrm{MHz}$. In this way, trapping of all other stable isotopes of strontium can be obtained simply by shifting the frequency of the main source (see Fig. 6a). With the addition of a repumper at $497 \mathrm{~nm}$ (resonant with ${ }^{3} \mathrm{P}_{2}{ }^{-3} \mathrm{D}_{2}$ ), typically we are able to load up to $3 \times 10^{8}$ atoms into the MOT in $160 \mathrm{~ms}$. The effect of the repumper light and optical collimation on the MOT loading is also reported in Fig. 6 b.

After the first stage cooling, the blue MOT is switched off and the atoms are transferred into a red MOT operated on the ${ }^{1} \mathrm{~S}_{0}-{ }^{3} \mathrm{P}_{1}$ intersystem crossing transition at $689 \mathrm{~nm}$. To this end we employ a slave laser injection-locked to the stable master ECDL. The red beam is overlapped to the blue beam on a dichroic mirror and from this point the two beams share the same broadband optics (mirrors, cubes, waveplates) up to the atoms. The recapture process involves a $200 \mathrm{~ms}$ long broadband phase to capture and further cool the atoms after which they are held in a single-frequency MOT which lasts for $40 \mathrm{~ms}$ [4]. During this last phase the red MOT beam intensity is decreased in order to further cool the atoms. Fig. 7 presents a typical time of flight measurement of the number of atoms trapped in the red MOT and their temperature, as a function of the total MOT beam intensity. Typically, we can trap $5 \times 10^{5}\left(4 \times 10^{6}\right.$ with repumper beam) ${ }^{88} \mathrm{Sr}$ atoms at $1 \mu \mathrm{K}$. The maximum transfer efficiency of the atoms from the blue MOT to the red MOT is

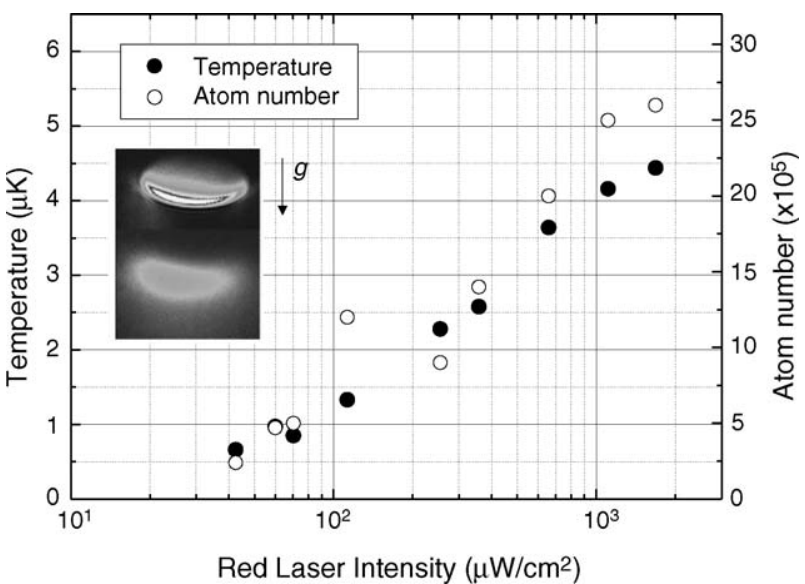

Fig. 7. Time of flight measurement of the temperature and number of ${ }^{88} \mathrm{Sr}$ atoms trapped in the red MOT as a function of the total trapping beam intensity. These data are taken without the use of the repumper light during the capture in the blue MOT. The inset shows a typical images of the atom cloud respectively after $200 \mu \mathrm{s}$ and $12 \mathrm{~ms}$ from the release of the atoms from the trap.

about $20 \%$, and the minimum temperature we have reached is $350 \mathrm{nK}$.

Finally, the cloud is transferred into the crossed beam FORT realized by focusing the two 922 -nm laser beams on the center of the trap. The transfer efficiency of the MOT to the crossed trap was found to be $10 \%$ with a maximum number of $2 \times 10^{588} \mathrm{Sr}$ atoms at $2 \mu \mathrm{K}$. At this wavelength, setting the optical-field polarization orthogonal to the magnetic field, the differential Stark shift between the ${ }^{1} \mathrm{~S}_{0}$ and ${ }^{3} \mathrm{P}_{1}$ levels is small with respect to the transition linewidth, allowing precision spectroscopy and laser cooling even on optically confined atoms [15]. At this point, the density is high enough that collision physics (two body, three body and light assisted collisions) become the dominant mechanism controlling temperature, density, trapping lifetimes, etc.

With a slight modification to the loading procedure, we are able to load multiple isotopes simultaneously [13]. We start to accumulate ${ }^{88} \mathrm{Sr}$ and ${ }^{86} \mathrm{Sr}$ in the ${ }^{3} \mathrm{P}_{2}$ magnetic trappable state [18]. Then the blue laser is turned off and the repumper is flashed on for a few ms. This puts the atoms back in their singlet ground state where we proceed with the second-stage cooling, recaptur-

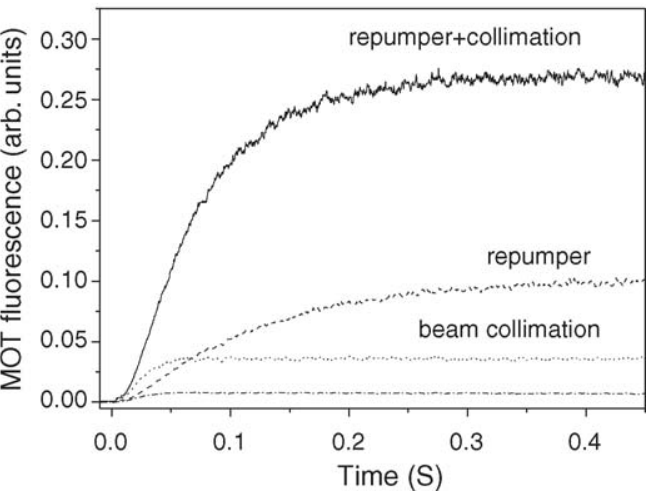

Fig. 6. (a) Blue MOT fluorescence signals, the four peaks refer to the four stable strontium isotopes ${ }^{88} \mathrm{Sr}(82.6 \%),{ }^{87} \mathrm{Sr}(7.0 \%),{ }^{86} \mathrm{Sr}(9.8 \%)$ and ${ }^{84} \mathrm{Sr}(0.56 \%)$; the inset shows the vertical magnification close to the laser frequency for ${ }^{84} \mathrm{Sr}$ trapping. (b) Loading curve for ${ }^{88} \mathrm{Sr}$ MOT in presence of repumper and optical beam collimation. 
ing them into a red double MOT operated with two slave lasers tuned to the different isotopes. During the last $50 \mathrm{~ms}$ of MOT operation the dipole trap laser is superimposed to the MOT allowing the transfer of the mixture in the dipole trap. In order to increase the transfer efficiency from the MOT to the FORT, we use a different geometry for the FORT. The dipole trap is realized by a single $922 \mathrm{~nm}$ beam focused in the center of the MOT with a waist of $w=15 \mu \mathrm{K}$. The potential trap depth obtained is $90 \mu \mathrm{K}$, and the radial (axial) oscillation frequencies are $2 \mathrm{kHz}(260 \mathrm{~Hz})$. We see no deleterious effect of the mixed species on the trap loading phase resulting in maximum transfer efficiency from red MOT of $40 \%$. Typically, we load the FORT with up to $3 \times 10^{5}\left(10^{6}\right)$ atoms at $15 \mu \mathrm{K}(20 \mu \mathrm{K})$ for the individual ${ }^{86} \mathrm{Sr}\left({ }^{88} \mathrm{Sr}\right)$ isotope. In the dipole trap we are able to measure most of the collisional properties of the sample (elastic and inelastic) as they relate to both inter and intra species [19].

\section{Conclusion}

We presented the laser sources and the experimental apparatus used for precision spectroscopy of intercombination lines of strontium at the $10^{-11}$ level and for preparing ultracold mixtures of atomic strontium. The setup allows us to trap more than $10^{6}$ $\left(10^{5}\right){ }^{88} \mathrm{Sr}\left({ }^{86} \mathrm{Sr}\right)$ atoms at $20 \mu \mathrm{K}(15 \mu \mathrm{K})$ at maximum phase space densities of 0.01 . This setup represents the first step in order to obtain a strontium BEC and to build a new optical frequency reference based on optical ultranarrow strontium lines.

While the ultimate performance of an optical clock working on the ${ }^{1} \mathrm{~S}_{0}-{ }^{3} \mathrm{P}_{1}$ transition for the most abundant ${ }^{88} \mathrm{Sr}$ isotope is limited by the linewidth of the transition (stability $10^{-14}$ at 1 s, accuracy $10^{-15}[20]$ ), a clock working on the doubly forbidden ${ }^{1} \mathrm{~S}_{0}-{ }^{3} \mathrm{P}_{0}$ transition could overcome the limitations both in stability and accuracy.

All the light sources used in our experiment are solid state; the compactness and reliability of such sources represent one of the first steps towards the realization of a transportable source of ultracold atoms to be employed in fundamental and applied physics on Earth and in space.

\section{Acknowledgements}

This work was supported by LENS, CRF, ASI, INFN, MIUR, INFM.

\section{References}

[1] H. Katori, M. Takamoto, V.G. Pal'chikov, V.D. Ovsiannikov, Phys. Rev. Lett. 91 (2003) 173005.

[2] A. Derevianko, S.G. Porsev, S. Kotochigova, E. Tiesinga, P.S. Julienne, Phys. Rev. Lett. 90 (2003) 063002.

[3] Y. Bidel, B. Klappauf, J.C. Bernard, D. Delande, G. Labeyrie, C. Miniatura, D. Wilkowski, R. Kaiser, Phys. Rev. Lett. 88 (2002) 203902.

[4] H. Katori, T. Ido, Y. Isoya, M. Kuwata-Gonokami, Phys. Rev. Lett. 82 (1999) 1116

[5] T. Ido, Y. Isoya, H. Katori, Phys. Rev. A 61 (2000) 061403.

[6] M. Takamoto, F.-L. Hong, R. Higashi, H. Katori, Nature 435 (2005) 03541 .

[7] T. Hong, C. Gramer, W. Nagourney, E.N. Fortson, Phys. Rev. Lett. 94 (2005) 050801.

[8] R. Santra, E. Arimondo, T. Ido, C.H. Greene, J. Ye, Phys. Rev. Lett. 94 (2005) 173002

[9] H. Marion, F. Pereira Dos Santos, M. Abgrall, S. Zhang, Y. Sortais, S. Bize, I. Maksimovic, D. Calonico, J. Grünert, C. Mandache, P. Lemonde, G. Santarelli, Ph. Laurent, A. Clairon, C. Salomon, Phys. Rev. Lett. 90 (2003) 150801

[10] R.W.P. Drever, et al., Appl. Phys. B 31 (1983) 97.

[11] J.K. Crane, M.J. Shaw, R.W. Presta, Phys. Rev. A 49 (1994) 1666.

[12] B. Couillaud, T.W. Hänsch, Opt. Commun. 35 (1981) 441.

[13] N. Poli, R.E. Drullinger, G. Ferrari, J. Léonard, F. Sorrentino, G.M. Tino, Phys. Rev. A 71 (2005) 061403(R).

[14] K. R. Vogel, Ph.D. Thesis, University of Colorado (1999).

[15] T. Ido, H. Katori, Phys. Rev. Lett. 91 (2003) 053001.

[16] G. Ferrari, P. Cancio, R. Drullinger, G. Giusfredi, N. Poli, M. Prevedelli, C. Toninelli, G.M. Tino, Phys. Rev. Lett. 91 (2003) 243002.

[17] G. Ferrari, T. Brzozowski, R. Drullinger, N. Poli, M. Prevedelli, C. Toninelli, G.M. Tino, Proc. SPIE 5478 (2004) 210.

[18] S.B. Nagel, C.E. Simien, S. Laha, P. Gupta, V.S. Ashoka, T.C. Killian, Phys. Rev. A 67 (2003) 011401.

[19] G. Ferrari, F. Sorrentino, N. Poli, R.E. Drullinger, G.M. Tino, PRL (2005), submitted for publication.

[20] Values are calculated assuming a quantum projection noise limited measurement done with $10^{6}$ free falling atoms at $400 \mathrm{nK}$ with a cycle time of $350 \mathrm{~ms}$. 